\title{
SOME BOUNDARY PROPERTIES OF FUNCTION ALGEBRAS
}

\section{H. S. BEAR ${ }^{1}$}

1. Introduction. Let $K$ be the closed unit disc in the complex plane, $U$ be the interior of $K$, and $\Gamma$ be the unit circle. Let $a$ be the algebra of all continuous complex valued functions on $K$ which are analytic on $U$. If $Q \mid \Gamma$ is the algebra of restrictions to $\Gamma$ of functions in $Q$, then $a \mid \Gamma$ is a maximal closed subalgebra of $C(\Gamma)$ (all continuous complex valued functions on $\Gamma$ ) [8]. In this note we investigate to what extent some of the classical boundary properties of analytic functions generalize to arbitrary function algebras, and in particular to maximal algebras. Specifically we shall look at possible generalizations of the following three theorems.

I. Fatou [4]: If $E$ is a closed subset of $\Gamma$ of measure zero, then there is some $f \in a$ which is zero exactly on $E$.

II. F. and M. Riesz [6]: If $f \in Q$ and $f=0$ on a subset $E$ of $\Gamma$ of positive measure, then $f \equiv 0$.

III. Rudin [7]: If $E$ is a closed subset of $\Gamma$ of measure zero and $f$ is any continuous function on $E$, then there is some function in $a$ which agrees with $f$ on $E$.

2. Assumptions. Let $A$ be a proper closed (uniform norm) subalgebra of $C(X)$ for some compact Hausdorff space $X$. Assume that $A$ separates points of $X$ and contains the constant functions. Assume also that $A$ is an essential algebra on $X$; i.e., that $A$ contains no proper closed ideal of $C(X)$ [1]. Let $\Sigma$ be the maximal ideal space of $A$ and assume that $X$ is a proper subset of $\Sigma$. We will consider the functions in $A$ as being defined on $\Sigma$ and use $A \mid X$ for the restrictions to $X$, and in general $A \mid E$ for the restrictions to any closed set $E$ in $\Sigma$ or $X$. A zero set is any set of the form $\{x \in \Sigma: f(x)=0\}$ for some nonconstant $f \in A$. A maximum set is any set of the form $\{x \in \Sigma: f(x)=\|f\|\}$ for some nonconstant $f \in A$. Every maximum set is obviously a zero set. A representing measure will be a positive regular Borel measure on $X$ such that for some $x \in \Sigma \sim X$ and every $f \in A, \int f d m=f(x)$. There is a representing measure corresponding to each point of $\Sigma \sim X[2]$.

For the example $Q$ of continuous analytic functions, the maximum sets and zero sets contained in $\Gamma$ are exactly those closed sets of measure zero (Theorems I and II above and Lemmas 1 and 2 of [7]). The representing measures are the Poisson measures on $\Gamma$.

Received by the editors November 8, 1958 and, in revised form, April 2, 1959.

1 This research was supported by a grant from the National Science Foundation. 


\section{Some boundary theorems.}

Lemma 1. If $E$ is a maximum set of $A$, then there is some $g \in A$ such that $g(x)=\|g\|=1$ if $x \in E$, and $|g(x)|<1$ if $x \notin E$.

Proof. Since $E$ is a maximum set, there is some $f \in A$ such that $E=\{x: f(x)=\|f\|=1\}$. Let $g=(1 / 2)(1+f)$; then $g \in A$, $E=\{x: g(x)=\|g\|=1\}$, and $|g(x)|<1$ if $x \in E$.

THEOREM 1. If $E$ is a maximum set for $A$, then $A \mid E$ is a closed subalgebra of $C(E)$.

Proof. ${ }^{2}$ If $J=\{f \in A: f=0$ on $E\}$, then $J$ is a proper closed ideal of $A$. It follows [5, p. 68] that $A / J$ is a Banach algebra in the quotient norm: $\|(f)\|=\inf \{\|g\|: g \in(f)\}$, where $(f)$ is the coset of $A / J$ containing $f$. Since $A \mid E$ and $A / J$ are isomorphic in an obvious way, we obtain our result by showing that the quotient norm is the sup norm in $A \mid E$. Let $f$ be any function in $A, \epsilon$ any positive number, and $g$ a function in $A$ such that $g(x)=1$ if $x \in E$, and $|g(x)|<1$ if $x \in E$. For sufficiently large $n,\left\|g^{n} f\right\|<\epsilon+\sup \{|f(x)|: x \in E\}$. Since $g^{n} f \in(f)$, we conclude that the quotient norm for $(f)$ is the sup norm for $f \mid E$.

The corollary below gives an extension of Rudin's Theorem III to a general maximal function algebra.

Corollary. If $A \mid X$ is an essential maximal closed subalgebra of $C(X)$, and $E$ is a maximum set for $A$, then $A \mid E=C(E)$.

Proof. If $A \mid X$ is an essential maximal subalgebra of $C(X)$, then $A \mid E$ is uniformly dense in $C(E)$ for every proper closed subset $E$ of $X$ [1]. Since $A \mid E$ is dense and closed in $C(E), A \mid E=C(E)$.

The next theorem and corollary provide a partial converse of Theorem 1, and in the case of the algebra $a$ exactly the converse of Rudin's theorem.

TheOREM 2. If $E$ is a closed subset of $\Sigma$ and $A \mid E=C(E)$, and $x_{0} \in \Sigma \sim E$, then there is some $f \in A$ such that $f=0$ on $E$ and $f\left(x_{0}\right) \neq 0$.

Proof. Suppose that every function in $A$ which is zero on $E$ is zero at $x_{0}$. Then for every $f \in A, f\left(x_{0}\right)$ is uniquely determined by $f \mid E$, and we can talk unambiguously of $f\left(x_{0}\right)$ for every $f \in A \mid E$ $=C(E)$. Thus evaluation at $x_{0}$ provides a (necessarily continuous [5, p. 69]) homomorphism of $C(E)$. This contradicts the facts that any homomorphism of $C(E)$ can be realized as evaluation at a point of $E$, and $A$ separates points of $\Sigma$.

2 Theorem 1 is due to J. L. Kelley and replaces a weaker form of the theorem discovered by the author. 
Corollary. If $A \mid E=C(E)$, then $E$ is contained in a zero set.

In Theorems 1 and 2 we replaced the notion of zero measure on the unit circle with the ideas of zero set and maximum set of the algebra. The next two theorems relate the zero sets and maximum sets with the Poisson-like representing measures. In the algebra $Q$, a subset $E$ of $\Gamma$ has measure zero if and only if $m(E)=0$ for every representing measure. Theorems 3 and 4 can be regarded as generalizations of the Riesz Theorem of $\S 1$.

THEOREM 3. If $E$ is a maximum set for $A$, and $E \subset X$, then $m(E)=0$ for every representing measure $m$.

Proof. Let $m$ represent the point $x_{0} \in \Sigma \sim X$, so that $f\left(x_{0}\right)=\int_{X} f d m$ for all $f \in A$. Let $g \in A, g(x)=1$ if $x \in E$, and $|g(x)|<1$ if $x \in \Sigma \sim E$. Then $g^{n}\left(x_{0}\right) \rightarrow 0$. On the other hand, the functions $g^{n}$ converge pointwise on $X$ to the characteristic function of $E$, so $g^{n}\left(x_{0}\right)=\int_{X} g^{n} d m$ $\rightarrow m(E)$.

We note that if $A$ is an essential maximal algebra on $X$, the condition $E \subset X$ is automatic for a maximum set $E$ by [2].

Let $C_{R}(X)$ denote all continuous real valued functions on $X$.

THEOREM 4. If the real parts of functions in $A$ are uniformly dense in $C_{R}(X)$, and $m$ represents $x_{0} \in \Sigma \sim X$, and $m(E) \neq 0$, and $f \in A$, and $f=0$ on $E$, then $f\left(x_{0}\right)=0$.

Proof. (A modification of the proof of the Riesz theorem given in $[3$, p. 50].)

If $m(E)=1$, then it is clear that $f\left(x_{0}\right)=0$, so we assume that $0<m(E)<1$. Let $\epsilon$ be positive, and $B$ so large that $\|f\| \exp \{-B /[1-m(E)]\}<\epsilon$. Let $\phi(x)=B / m(E)$ if $x \in E$, and $\phi(x)$ $=-B /[1-m(E)]$ if $x \in X \sim E$. Then $\int \phi d m=0$. Let $V$ be a neighborhood of $E$ and $w$ a continuous real function on $X$ which equals $\phi$ on $E$ and off $V$, and takes intermediate values on $V \sim E$. Note that $\int w d m=\int(w-\phi) d m=\int_{V \sim E}(w-\phi) d m$. We can therefore choose $V$ with $m(V \sim E)$ so small that $\left|\int w d m\right|<\epsilon$. In addition we require that $m(V \sim E)$ be so small that $\int_{V \sim E}|f| e^{w} d m<\epsilon$. By the choice of $B$, we have $\int_{X \sim V}|f| e^{w} d m<\epsilon$. Now let $u$ be the real part of a function in $A$ with $u$ close enough to $w$ that $\left|u\left(x_{0}\right)\right|=\left|\int u d m\right|<\epsilon$ and $\int_{X \sim E}|f| e^{u} d m$ $<2 \epsilon$. Let $u+i v \in A$, with $v\left(x_{0}\right)=0$, and let $g=e^{u+i v}$. Then $g \in A$, $|g|=e^{u}$, and $g\left(x_{0}\right)=e^{u\left(x_{0}\right)}>e^{-\epsilon}$. Hence

$$
\begin{aligned}
\left|f\left(x_{0}\right)\right| & \leqq\left|f\left(x_{0}\right)\right| g\left(x_{0}\right) e^{\epsilon}=e^{\epsilon}\left|\int f g d m\right| \leqq e^{\epsilon} \int|f||g| d m \\
& =e^{\epsilon} \int|f| e^{u} d m=e^{\epsilon} \int_{X \sim E}|f| e^{u} d m \leqq 2 \epsilon e^{\epsilon} .
\end{aligned}
$$


Since $\epsilon$ is arbitrary, $f\left(x_{0}\right)=0$.

Corollary. If the real parts of functions in $A$ are dense in $C_{R}(X)$, and $m(E)>0$ for every representing measure $m$, then every function in $A$ which is zero on $E$ is identically zero.

Corollary. If the real parts of functions in $A$ are uniformly dense in $C_{R}(X)$, and there is a zero set $F$ such that $E \subset F \subset X$, then $m(E)=0$ for every representing measure $m$.

Comment. The fact which is conspicuously missing from these theorems is some analogue of Fatou's Theorem I. For example, the last corollary above assumes that $F$ is a zero set (in $\Sigma$ ) instead of a set of the form $\{x \in X: f(x)=0\}$.

\section{REFERENCES}

1. H. S. Bear, Complex function algebras, Trans. Amer. Math. Soc. vol. 90 (1959) pp. 383-393.

2. - A strong maximum modulus theorem for maximal function algebras, Trans. Amer. Math. Soc. vol. 92 (1959) pp. 465-469.

3. C. Caratheodory, Theory of functions of a complex variable, vol. 2, New York, 1954.

4. P. Fatou, Series trigonométriques et séries de Taylor, Acta Math. vol. 30 (1906) pp. $335-400$.

5. L. H. Loomis, An introduction to abstract harmonic analysis, New York, 1953.

6. F. and M. Riesz, Über die Randwerte einer analytischen Funktion, Quatrième Congrès des Math. Scand., 1916, pp. 27-44.

7. Walter Rudin, Boundary values of continuous analytic functions, Proc. Amer. Math. Soc. vol. 7 (1956) pp. 808-811.

8. John Wermer, On algebras of continuous functions, Proc. Amer. Math. Soc. vol. 4 (1953) pp. 866-869.

UNIVERSITY OF WASHINGTON 